\title{
X\#Ray Emission from Hot Gas in Galaxy Mergers
}

\section{Citation}

Cox, T. J., Tiziana Di Matteo, Lars Hernquist, Philip F. Hopkins, Brant Robertson, and Volker Springel. 2006. "X\#Ray Emission from Hot Gas in Galaxy Mergers." The Astrophysical Journal 643 (2): 692-706. https://doi.org/10.1086/503284.

\section{Permanent link}

http://nrs.harvard.edu/urn-3:HUL.InstRepos:41381797

\section{Terms of Use}

This article was downloaded from Harvard University's DASH repository, and is made available under the terms and conditions applicable to Other Posted Material, as set forth at http:// nrs.harvard.edu/urn-3:HUL.InstRepos:dash.current.terms-of-use\#LAA

\section{Share Your Story}

The Harvard community has made this article openly available.

Please share how this access benefits you. Submit a story.

\section{Accessibility}


Draft version August 30, 2018

Preprint typeset using $\mathrm{LATEX}_{\mathrm{E}}$ style emulateapj v. 6/22/04

\title{
X-RAY EMISSION FROM HOT GAS IN GALAXY MERGERS
}

\author{
T. J. Cox ${ }^{1}$, Tiziana Di Matteo ${ }^{2}$, Lars Hernquist ${ }^{1}$, Philip F. Hopkins ${ }^{1}$, Brant Robertson ${ }^{1}$, And Volker Springel $^{3}$ \\ Draft version August 30, 2018
}

\begin{abstract}
We examine X-ray emission produced from hot gas during collisions and mergers of disk galaxies. To study this process, we employ simulations that incorporate cosmologically motivated disk-galaxy models and include the effects of radiative cooling, star formation, supernova feedback, and accreting supermassive black holes. We find that during a merger, the colliding gas in the disks is shock-heated to X-ray-emitting temperatures. The X-ray luminosity is spatially extended, rises during the initial stages of the merger, and peaks when the galactic centers coalesce. When a physical model for accreting black holes is included, the resulting feedback can drive powerful winds that contribute significantly to the amount and metallicity of hot gas, both of which increase the X-ray luminosity. In terms of their stellar kinematics and structural properties, the merger remnants in our simulations resemble elliptical galaxies. We find that the X-ray luminosities of the remnants with $B$-band luminosities in the range $L_{B} \sim 10^{10}-10^{11} L_{\odot}$ are consistent with observations, while remnants with smaller or larger masses are underluminous in X-rays. Moreover, because the majority of the merger remnants are broadly consistent with the observed scaling relations between temperature, $B$-band luminosity and X-ray luminosity we conclude that major mergers are a viable mechanism for producing the X-ray halos of large, luminous elliptical galaxies.

Subject headings: galaxies:active — galaxies:evolution — galaxies:formation — galaxies:interactions — methods:N-body simulations — X-rays: galaxies
\end{abstract}

\section{INTRODUCTION}

It is now well established that elliptical galaxies contain substantial quantities of hot gas (see Mathews \& Brighenti 2003, and references therein). Detailed studies of the X-ray emission produced by this hot gas have revealed that its luminosity is correlated with the gas temperature, B-band luminosity, and stellar velocity dispersion of the host elliptical galaxy (O'Sullivan. Forbes. \& Ponman 2001a; O'Sullivan. Ponman. \& Collins 2003). These correlations argue for an evolutionary link between the process that formed the elliptical galaxy and the surrounding, $\mathrm{X}$-ray emitting gas.

According to the "merger hypothesis," elliptical galaxies are formed when two spiral galaxies interact and merge (Toomre \& Toomre 1972; Toomre 1977). Thus, it is possible that disk-galaxy interactions transform spirals galaxies into ellipticals and generate their hot gaseous halos. Using the X-ray satellite ROSAT, Read \& Ponman (1998) investigated the X-ray luminosity evolution along the "Toomre sequence" (Toomre 1977), a series of eight local galaxies representing different stages of merging, in chronological order. Their study found that extended $\mathrm{X}$-ray emission is produced during the encounter and persists as the nuclei merge. Similar results obtain from observations of merging and interacting systems using the higher resolution ChandraX - rayObservatory (Fabbiano et al. 2001; McDowell et al. 2003; Huo et al. 2004).

A puzzling aspect of the analysis of Read \& Ponman

1 Harvard-Smithsonian Center for Astrophysics, 60 Garden Street, Cambridge, MA 02138, USA

2 Carnegie-Mellon University, Dept. of Physics, 5000 Forbes Ave., Pittsburgh, PA 15213, USA

3 Max-Planck-Institut für Astrophysik, Karl-SchwarzchildStraße 1, 85740 Garching bei München, Germany
(1998) is that the merger remnants appear to be underluminous when compared to typical elliptical galaxies, bringing into question the likelihood that merger remnants evolve into normal ellipticals. These authors suggested several methods by which a merger remnant could acquire an X-ray halo, late infall of tidal material, reacquisition of gas ejected by galactic winds during the merger, and ongoing mass-loss by stars born during a merger-induced starburst. One clue to the origin of the hot gas lies in the correlation between the X-ray and $B$-band luminosities. O'Sullivan et al. (2001a), also using $R O S A T$, found that $L_{X} \propto L_{B}$ for less massive ellipticals, consistent with their X-ray luminosity being primarily stellar in origin. However, the X-ray emission from more luminous ellipticals varies as $L_{X} \propto L_{B}^{2}$, indicating a non-stellar formation process.

Another clue to the origin of hot gas in spheroidal systems is its high metallicity. Humphrev \& Buote (2006) analyzed a sample of 29 X-ray luminous elliptical galaxies and found that their two-phase models for the hot Xray emitting plasma yielded metallicities that were typically solar or even higher and generally correlated with the stellar metallicity. This result indicates that the gas that makes up the hot coronae surrounding ellipticals has been recycled from star-forming gas and likely once resided within the dense central regions of the galaxies. This also suggests that a significant amount of energy was injected into this gas in order to expel it from the depths of the potential well and redistribute it throughout the galactic halo.

The work described here is a first attempt to address the viability of galaxy mergers in generating X-ray emitting gaseous halos consistent with observed ellipticals. While numerical simulations have demonstrated that galaxy interactions can indeed produce remnant galaxies whose stellar component is reminiscent of local ellipticals 
(Barnes 1992; Barnes \& Hernquist 1996; Hernquist 1992 , 1993b; Naab \& Burkert 2003; Robertson et al. 2005; Springel et al. 2005a; Cox et al. 2005a), little is known theoretically about the evolution of the hot gas component in these events. Barnes \& Hernquist (1996) considered simulations in which the gas was not allowed to cool radiatively and showed that in this limit it would be mostly shock heated to the virial temperature of the remnant, leaving a corona of hot, X-ray emitting material. Later, Cox et al. (2004) showed that the amount of hot gas is dependent on the interaction orbit, i.e., more radial encounters produce more hot gas. Yet the ability of this hot gas to produce X-rays, the luminosity of the $\mathrm{X}$-ray emission, and the correlation to the remnant stellar properties are all open questions. In particular, these studies did not attempt to model all feedback processes in detail. Recently, Springel. Di Matteo. \& Hernquist (2005b) have developed methods for modeling feedback from both star formation and black hole growth in galaxy mergers. This addition to our galaxy modeling has significant implications for the X-ray evolution as the feedback energy from an accreting black hole can generate a substantial amount of hot gas through the production of a large-scale galactic wind.

The rest of this paper is organized as follows. In $\S 2$ we describe the numerical simulations upon which are results are based. In $\S[$ we detail the X-ray emission produced during and after the collision of two gas-rich disk galaxies. We follow this by showing how the global properties of X-rays and hot gas depend on progenitor mass and gas fraction in $\S 4 \S 5$ discusses our results, specifically addressing the X-ray luminosities of our merger remnants and the generation of hot gaseous halos observed in luminous elliptical galaxies. Finally, we conclude in $\$ 6$

\section{MERGER SIMULATIONS}

To simulate mergers of disk galaxies, we employ GADGET2 (Springel 2005), which is based on a "conservativeentropy" formulation (Springel \& Hernquist 2002) of smoothed particle hydrodynamics $(\mathrm{SPH})$ that conserves both energy and entropy (unlike earlier versions of SPH; see e.g., Hernquist 1993a), while improving shockcapturing. The code includes the effects of radiative cooling and heating by a uniform UV background, and star formation, supernova feedback, and metal enrichment are treated in the manner detailed in Springel \& Hernquist (2003). Within this formalism, the sub-resolution physics of star formation and supernova feedback acts to maintain star-forming gas at a temperature specified by an effective equation of state (see e.g., Robertson et al. 2004). As an extension of this star formation and feedback model, Springel et al. (2005b) introduced an additional parameter $q_{\mathrm{EOS}}$, so that the effective equation of state can be varied between that for an isothermal gas at $T_{\text {eff }}=10^{4} \mathrm{~K}, q_{\text {EOS }}=0$, and the "stiff" Springel-Hernquist equation of state, which has an effective temperature $T_{\mathrm{eff}} \approx 10^{5} \mathrm{~K}, q_{\mathrm{EOS}}=1$. In the work presented here, $q_{\mathrm{EOS}}$ was set to 0.25 resulting in a mass-weighted temperature of star-forming gas $\sim 10^{4.5} \mathrm{~K}$.

Our simulations also allow the presence of a supermassive black hole (BH). BHs are represented by "sink" particles that can accrete neighboring gas at a rate given by the Bondi-Hoyle-Lyttleton approximation with an imposed upper limit equal to the Eddington rate. A small fraction (typically 5\%) of the bolometric luminosity $\left(0.1 \dot{M} c^{2}\right.$, assuming an accretion efficiency of $\left.10 \%\right)$ thermally couples to the gas surrounding the BH. These parameters are selected so that simulations of disk-galaxy mergers successfully reproduce the normalization of the $M_{B H^{-}} \sigma$ relation (Di Matteo, Springel, \& Hernquist 2005). The BH, when included, has a seed mass of $\approx 10^{5} M_{\odot}$, although we find that the outcome is insensitive to this choice. A more complete discussion of the methodology is provided in Springel et al. (2005b).

The mergers used throughout this paper are identical to those in Cox et al. (2005a) and are described in more detail there and in Robertson et al. (2005). A full description of the construction of these galaxies is given in Springel et al. (2005b); here we simply outline the parameter choices for the simulations discussed in this work. The composite system contains an exponential disk embedded in a dark matter halo. For simplicity we do not include a spheroidal bulge. The dark-mater halo is initialized with a Hernquist (1990) profile, a concentration $c=9$, and a spin parameter $\lambda=0.041$. In Section 4 we investigate hot gas production for mergers between equal mass galaxies with a range of masses, but much of our discussion is based on a fiducial case where the progenitor galaxies have properties similar to the Milky Way. The virial velocity in this case is $V_{200}=160 \mathrm{kms}^{-1}$, resulting in a halo mass of $1.4 \times 10^{12} M_{\odot}$. The exponential disk composes $4.1 \%$ of the total mass, with a fixed fraction $f$ in a collisional gaseous component. Our fiducial simulation fixes $f=0.4$. The compound galaxy is realized with 500,000 particles to represent the dark matter and 50,000 to represent the disk. Since the disk comprises both stellar and gaseous components, a fraction $f \times 50,000$ are consider gas and the remainder represent the collisionless stellar disk.

Once the model galaxies are generated, we collide two disks by placing them on a parabolic orbit with an initial separation of $143 \mathrm{kpc}$ and a pericentric distance of $7.1 \mathrm{kpc}$, resulting in a nearly radial collision. To investigate the consequences of black hole growth and feedback, we have performed simulations that include centrally accreting BHs, and are labeled as such, and others that do not that are referred to as "standard". Figure 11 shows six representative images of both types of galaxy mergers (i.e., with and without BHs). Details of the morphology, dynamics and resulting star formation can be found elsewhere (see e.g., Barnes \& Hernquist 1996; Mihos \& Hernquist 1996; Springel 2000; Springel et al. 2005b; Cox et al. 2005b).

Figure 11 also demonstrates the additional diffuse gas present when the merger includes an accreting black hole. Owing to the energy put into the gas by the growing $\mathrm{BHs}$, particularly during the final merger, a significant fraction of the gas participates in a large-scale galactic wind. This "blowout" phase is very efficient and star formation is almost completely shut off (Springel et al. 2005a), leaving a remnant that quickly reddens and evolves nearly passively (Hopkins et al. 2006c). Moreover, as discussed further in the following section, the expulsion of the gas during the blowout greatly enhances the X-ray luminosity relative to cases in which no $\mathrm{BH}$ is present because 


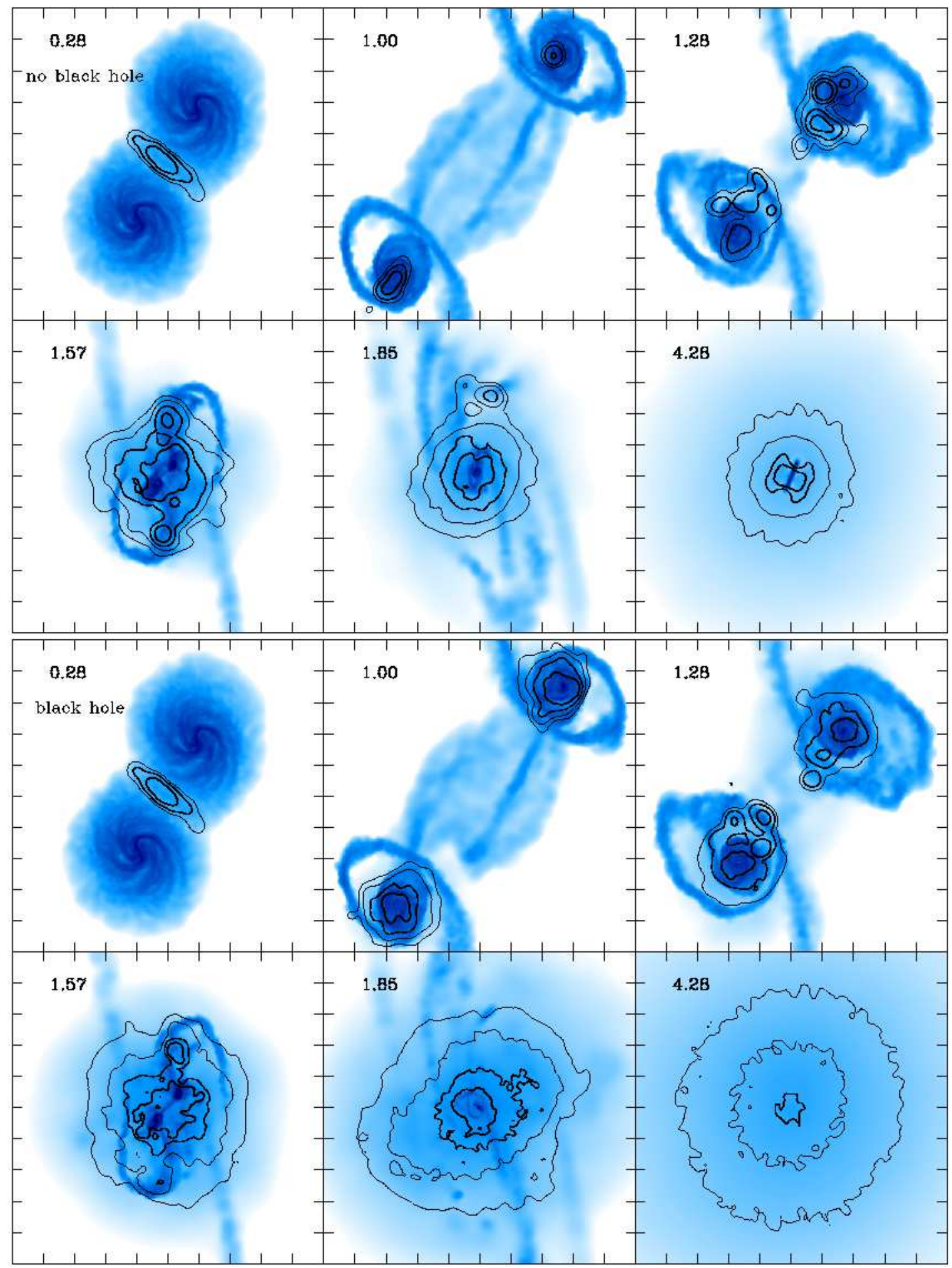

FIG. 1.- Time sequence of the projected gas density, shown in blue scale, with X-ray contours overlaid (in black). The top six panels demonstrate the collision for our standard, no-black hole merger, while the bottom six panels are the case in which a central supermassive black hole is included. Each panel is $100 \mathrm{kpc}$ square and the color and X-ray contour scales are identical. The simulation time, in Gyr, is indicated in the top left of each plot, and the merger occurs at $T \approx 1.6 \mathrm{Gyr}$. 
not only is there additional gas throughout the halo, but this gas is highly metal-enriched.

While a complete survey of the parameter space that describes the merger of two disk galaxies is beyond the scope of this work, it is important to understand how the production of hot gas depends on our initial assumptions. To this end, we merge disk galaxies with varying gas fractions and masses and describe the outcomes in Section 4

\section{A CASE STUDY}

In this section we provide a detailed look at the evolution of hot gas and X-rays during one merger. While the X-ray luminosity, gas temperature and mass depend strongly on the initial halo size/mass and disk gas fraction (discussed in $\S$ ), the production of hot gas during a galaxy collision is a general feature of all our simulations.

\subsection{X-ray emission}

Our analysis of the simulations assumes that X-rays are produced by the cooling of hot, diffuse gas. In reality, the observed X-ray emission has contributions from stellar sources such as supernova remnants and X-ray binaries, as well as accreting black holes and the hot phase of the interstellar medium. Because the composite emission from these sources is generated mostly in the dense central regions of the galaxies, this luminosity may be heavily extinguished owing to the large column density of intervening gas and dust. In fact, as described by Hopkins et al. (2005d, 2006a), we find that the central accreting BHs in merger simulations remain obscured for the majority of their active lifetimes. To simplify our analysis, we restrict ourselves to X-ray emission from the diffuse hot gas since it should be relatively unaffected by obscuration.

We follow Navarro et al. (1995) and assume the X-ray luminosity for each SPH particle can be estimated from

$$
L_{\mathrm{X}, i}=1.2 \times 10^{-24}\left(\frac{m_{\mathrm{gas}, i}}{\mu m_{p}}\right) \frac{\rho_{i}}{\mu m_{p}}\left(\frac{k T_{i}}{\mathrm{keV}}\right)^{1 / 2} \operatorname{ergs~s}^{-1}
$$

where $m_{p}$ is the proton mass, $\mu$ is the mean molecular weight ( 0.6 for a fully ionized primordial plasma), and $m_{\text {gas }, i}, \rho_{i}$, and $T_{i}$ are the mass, density and temperature of the $i^{\text {th }}$ gas particle in cgs units, respectively. Equation (11) assumes that the primary mechanism for X-ray emission is thermal bremsstrahlung, an assumption consistent with the zero-metallicity cooling included in the simulation. However, metal enriched gas with a temperature of $\sim 10^{6} \mathrm{~K}$ cools primarily through metal-line emission, a mechanism that is more efficient and would thus produce a much larger X-ray luminosity. In this sense, the X-ray emission computed via Equation (1) is a lower limit.

In light of the increased X-ray luminosity expected from metal-enriched gas, we supplant equation (1) with X-ray emission calculated using a Ravmond \& Smith (1977, hereafter RS77) code that does include metal-line emission. The gas metallicity is provided by the starformation model (see Springel \& Hernquist 2003) assuming a metal yield of $2 \%$, and we include emission from 0.1 to $2 \mathrm{keV}$.

The bolometric luminosity is then calculated according

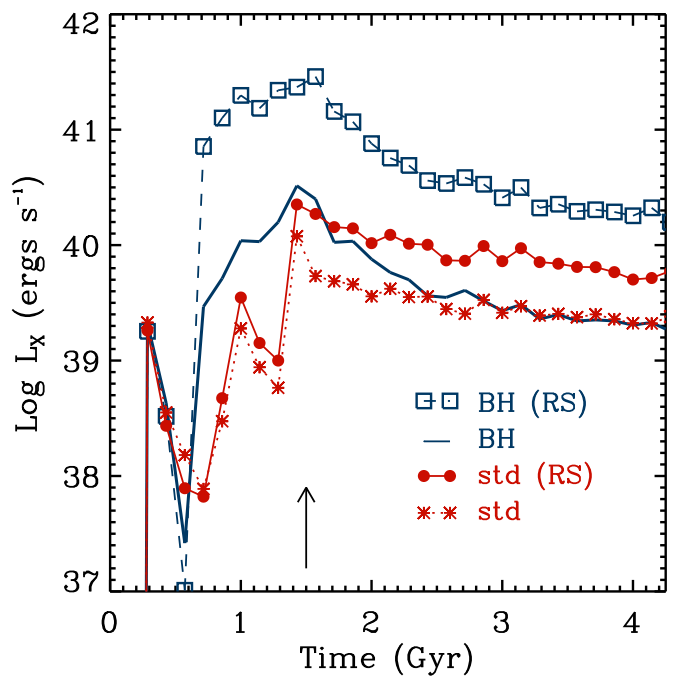

FIG. 2. - Bolometric X-ray luminosity owing to diffuse hot gas during simulated major mergers. Shown are mergers with ("BH", blue) and without ("std", red) accreting black holes. Each simulation has two curves, one, in which the gas is assumed to have zero metallicity (blue, solid line, and red,asterisks), and, the other, in which the emission is calculated using Ravmond \& Smith (1977, RS77) models, which include metal-line cooling (blue, open squares, and red,filled circles). The arrow at $T=1.5 \mathrm{Gyr}$ shows the time of the final merger in both simulations.

to

$$
L_{\mathrm{X}, \text { bolo }}=\sum_{i=1}^{N_{\text {hotgas }}} L_{\mathrm{X}, i}
$$

where the sum is carried out over all gas particles with density below the critical density for star formation $\rho_{\text {thresh }} \approx 10^{-2} M_{\odot} \mathrm{pc}^{-3}$ and a temperature greater than $10^{5.2} \mathrm{~K}$. Gas particles that meet these criteria are fully ionized (for zero metallicity) and are deemed "hot" or "X-ray" gas from hereafter. We note that our low temperature $\left(10^{5.2} \mathrm{~K}\right)$ threshold for gas to be included in the X-ray luminosity probes temperatures below current observational limits. However, this cut-off does serve as an accurate gauge of the amount of hot, ionized gas.

\subsection{Time evolution}

Figure 2 shows the bolometric X-ray luminosity as determined by equation (2) for the entire major merger. For both simulations, two X-ray luminosities are shown. One, where the X-ray luminosity is calculated via equation (1), in essence when the gas is assumed to have zero metallicity, and the second uses the RS77 code to include metal-line cooling. As expected, the inclusion of metallicity dependent cooling greatly enhances the X-ray emission produced during the galaxy merger. In addition, the spatial distribution of the X-ray emission is shown with overlaid contours in Figure 1

Initially, the X-ray emission from both galaxies is negligible because we ignore contributions from the starforming interstellar medium. X-ray emission begins when the gas disks first interact, in shocks that lie directly between the two disks (see $T=0.28$ Gyr panel in Fig. 1). In general, the majority of the X-ray emission is produced by shock-heated gas, and thus the X-ray 
emission essentially tracks shocks that occur during the galaxy merger.

Figure 11 also shows that when the galaxies separate, the X-ray emission is concentrated around the two galactic nuclei. This emission resembles that in observed systems that are in a similar dynamical state, such as the Mice (Read 2003). Figure 1 also demonstrates that when BHs are present, additional hot gas is generated by thermal feedback from $\mathrm{BH}$ accretion beginning at $T=0.7$ Gyr. This results in an $L_{X}$ that is over an order of magnitude larger than the no-BH case.

The excess X-ray emission from $T=0.7$ to 1.5 is generated by small-scale winds in the still-separated disks. These winds are, in general, collimated and escape perpendicular to the disk plane, similar to those shown in Springel et al. (2005b, their Fig. 9). In the simulations presented here, the winds are generated by the energy input by the accreting black holes and thus are present only in the $\mathrm{BH}$ simulations. Collimated winds such as these are seen in many starbursting systems (Martin 2005; Rupke et al. 2002; Veilleux et al. 2005; Strickland et al. 2002).

One of the most prominent features in Figure 2 is the coincidence of the peak X-ray luminosity with the final merger, regardless of whether or not BHs are present. This X-ray emission results from the abundant shocks that occur as the gas disks attempt to follow the collisionless stellar nuclei. Gas is heated to $10^{6}-10^{8} \mathrm{~K}$ and forced out of the central regions owing to shocks and pressure forces. Hence, the X-ray luminosity is spatially extended, as can be seen in Figure 1 from $T \sim 1.5-1.9$ Gyr.

The maximum X-ray emission ranges from $8 \times 10^{39}$ to $2.5 \times 10^{41}$ ergs s$^{-1}$, depending on the presence of a $\mathrm{BH}$ and if metallicity-dependent cooling is included. These luminosities are consistent with observations of the $\mathrm{X}$ ray emission seen in interacting and merging systems (Fabbiano et al. 2001; Xia et al. 2002; McDowell et al. 2003; Huo et al. 2004; Jenkins et al. 2005; Read 2005; Smith et al. 2005) as well as that expected on theoretical grounds (Jog \& Solomon 1992). Because the X-ray luminosity is a direct consequence of the collision between gas in each progenitor disk, we expect a range of peak luminosities depending on the gas content in each galaxy, their (relative and cumulative) mass and the merger orbit. The mass and gas fraction dependence of $L_{X}$ is investigated further in $\S 4$

After the galactic nuclei coalesce, at $T \sim 1.6 \mathrm{Gyr}$, the X-ray luminosity decreases. When no $\mathrm{BH}$ is present, $L_{X}$ drops slowly as gas settles into hydrostatic equilibrium, and tidal material slowly returns to the remnant. However, when an accreting $\mathrm{BH}$ is included, the drop in $L_{X}$ is more pronounced owing to the "blowout" phase which expels a significant amount of metal-enriched, Xray emitting gas. The eventual fate of gas caught up in the galactic wind is dictated by its energy relative to the depth of the galaxy potential well. In the simulation we describe in this section, a substantial amount of gas is injected with enough energy to completely unbind it from the remnant. However, even if a significant amount of gas is ejected from the system, the blowout does not leave the remnant completely devoid of gas. A substantial amount ( $\sim 75 \%)$ of the remaining gas remains bound to the remnant galaxy, is spread throughout the dark matter halo,
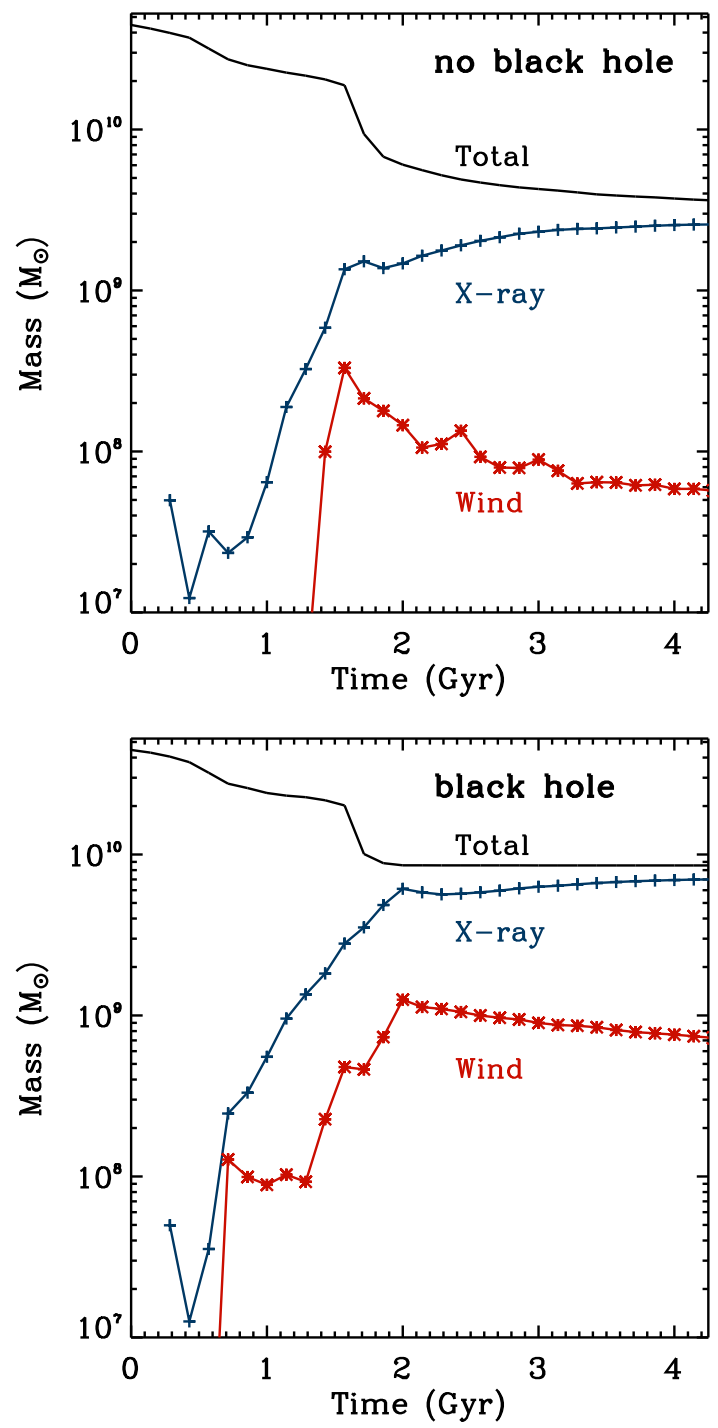

FIG. 3.- Time evolution of gas mass during the disk galaxy merger. Shown with a solid black line is the total gas mass. During the simulation, gas is steadily depleted owing to efficient star formation. The (blue) line with crosses is the mass of gas which emits X-rays. The (red) line with asterisks is mass of gas which becomes unbound from the system. Two plots are shown, one with and the other without a centrally accreting black hole.

and makes up the X-ray corona.

Figure 3 provides an accounting of the gas mass for mergers with and without accreting BHs. This figure also shows the mass of unbound (i.e., $E>0$ ) gas and the mass of gas that contributes to the X-ray luminosity. Gas that is not unbound or X-ray emitting is typically cold and, depending on its density, star forming. Consistent with the findings of Springel et al. (2005a) there is little cold gas in the remnant that includes a $\mathrm{BH}$ and star formation is efficiently quenched subsequent to the merger at $T \sim$ 1.6 Gyr.

Figure 3 demonstrates that the $\mathrm{BH}$ has a significant impact on the amount of X-ray and wind gas. Owing to the merger-induced energy input from the $\mathrm{BH}$, and the significant wind that ensues, the $\mathrm{BH}$ system has over 10 times the mass of unbound gas as compared to the no $\mathrm{BH}$ case. The $\mathrm{BH}$ feedback also contributes the $\mathrm{X}$-ray 
gas and results in nearly 3 times the amount of mass as compared to when no $\mathrm{BH}$ is present. At first the differing amounts of X-ray gas in the two cases seem at odds with the identical X-ray luminosity (for emission by thermal bremsstrahlung) demonstrated in Figure 2 However, as shown in $\S 3.5$ (see Fig. 8) the gas within the inner $10 \mathrm{kpc}$ of the remnant without a $\mathrm{BH}$ is over an order of magnitude denser than the no $\mathrm{BH}$ remnant and thus is more efficient at producing X-rays. We note that the thermal structure is quite similar between the two remnants, as is shown in $\S 3.4$

The hot gas in the remnant relaxes on a timescale that is short when compared to that of the stellar component of the halo, as reflected by both the regularity of the $\mathrm{X}$-ray isophotes (see the bottom-right panel in Fig. 11) and the steady X-ray luminosity after $T=3.0 \mathrm{Gyr}$, a little more than 1.5 Gyr after the merger. In contrast, stellar material in tidal tails is accreted by the remnant to produce long-lived shells, loops, and other fine structure (Hernquist \& Spergel 1992) by "phasewrapping" (Quinn 1984; Hernquist \& Quinn 1988, 1989). Similar features are seen around many ellipticals (Malin \& Carter 1983; Schweizer 1980; Schweizer et al. 1990). Because this debris is on nearly radial orbits, gas in the tails is stripped out and deposited in the halo or inner regions of the remnant (Hernquist \& Weil 1992; Weil \& Hernquist 1993), and the shells quickly redden.

The BH-driven wind is also efficient at dispersing metals. Because the blowout is triggered by the centrally located BH, gas that is ejected from the system has been significantly enriched by metals. To demonstrate this more explicitly we refer to Figure 4. Plotted are contours of the gas density at $T \approx 0.9 \mathrm{Gyr}$, shortly after the first passage of the two disks. Overlaid are (red) dots that denote the gas particles that will eventually constitute the hot X-ray emitting gaseous halo. When no $\mathrm{BH}$ is present, the gas in the X-ray halo was once almost exclusively composed of loosely bound tidal material. In these low-density regions, little star formation occurs and thus metal enrichment is only modest. While this same loosely bound tidal material will make it into the X-ray halo of the $\mathrm{BH}$ remnant, the majority of the X-ray gas in this case comes from gas that was once more centrally concentrated. This gas is spread throughout the disk and has been significantly enriched by star formation.

For the BH merger shown in Figure 4 X-ray gas in the remnant has an X-ray weighted mean metallicity of 0.9 solar. The unbound wind material has an even higher metallicity, nearly twice solar. In this sense, the wind could be considered "mass loaded." The high metallicity of the gas suggests that the wind is made up primarily of the densest, most metal-rich gas. In contrast, without the energy input from a $\mathrm{BH}$, the X-ray gas is $1 / 10 Z_{\odot}$, and the small amount of unbound gas is less than $1 / 20 Z_{\odot}$. Without the energy input from the $\mathrm{BH}$ essentially the entire metal budget is locked in stars or star-forming gas.

The dispersal of metals by the wind also has a profound influence on the X-ray emission. Because the metallicity of the X-ray-emitting gas is nearly 10 times larger in the $\mathrm{BH}$ remnant as compared to the standard remnant, the X-ray luminosity is greatly increased. We show the $\mathrm{X}$-ray luminosity when metal-line cooling is considered in Figure 2. We hereafter restrict our analysis to X-ray
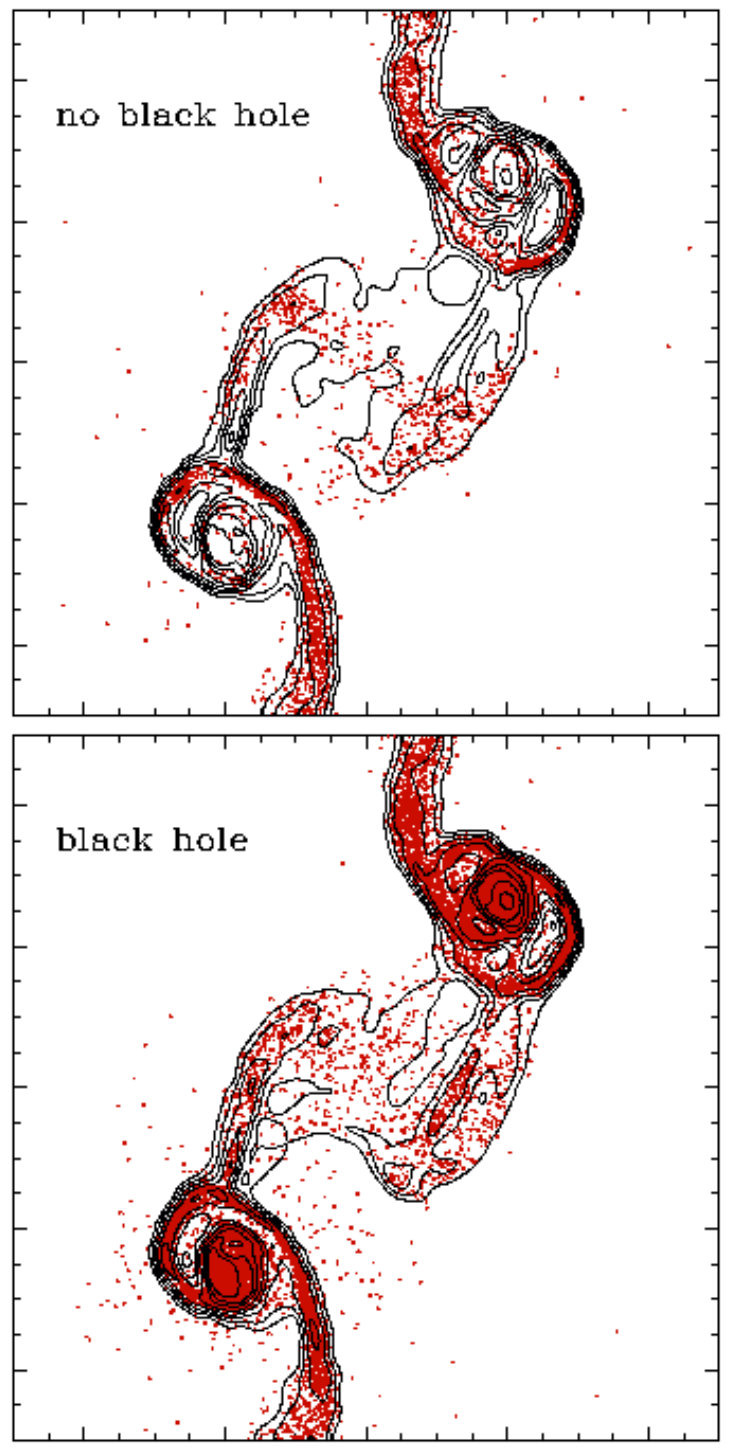

Fig. 4.- Contours show the gas density subsequent to the first passage of the interacting dark galaxies. Tidal tails and a bridge are clearly present. Overlaid are $(r e d)$ dots that denote gas particles that eventually end up as part of the hot, X-ray emitting gaseous halo. Two images are shown, one with and the other without a centrally accreting black hole.

emission calculated using the metal-line cooling of RS77.

\subsection{Time evolution of $L_{X} / L_{B}$}

Because galaxies encompass a range of masses, studies of the X-ray emission caused by galaxy interactions have typically normalized the X-ray emission by the $B$-band luminosity of the host galaxy (Read \& Ponman 1998; O'Sullivan et al. 2001b). In order to facilitate a more direct comparison to these studies, we show $L_{X} / L_{B}$ for our mergers in Figure [5] For simplicity, we ignore the effects of extinction when computing $L_{B}$. This approximation is likely to be valid during the post-merger phases but may dramatically overestimate the luminosity during the highly obscured merger stage. In any case, many of the features in $L_{X} / L_{B}$ are very similar to those of just $L_{X}$ (shown in Fig. 2). This result is not surprising given the moderate gas fraction of our progenitor disks, 


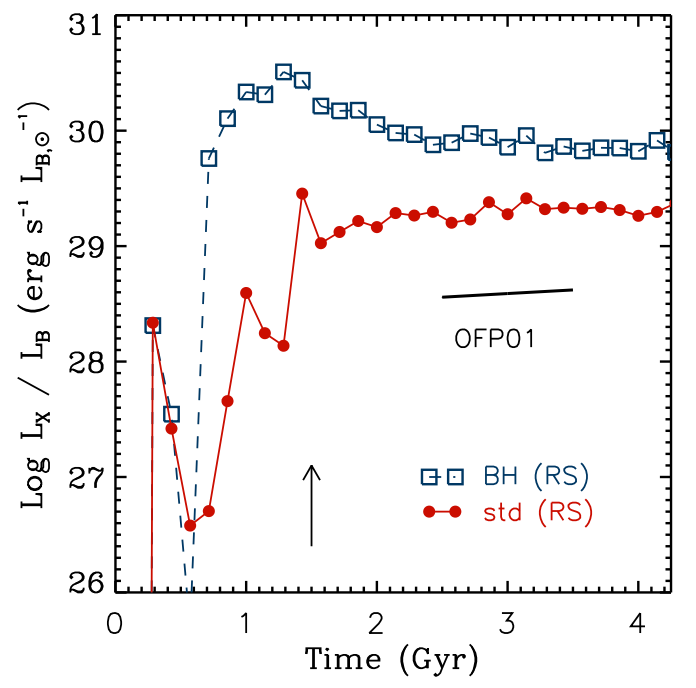

FIG. 5.- As in Fig. 2 except the X-ray luminosity has been divided by the $B$-band luminosity. For clarity, only the metal-line $\mathrm{X}$-ray emission is plotted. Again, the arrow at $T=1.5$ Gyr shows the time of the final merger in both simulations, and the slope shows the observed slope (O'Sullivan et al. 2001b, OFP01).

and hence the modest fraction of stellar mass that is produced during the merger. For the most part, $L_{B}$ slowly decreases over time owing to an aging stellar population.

Both $L_{X}$ and $L_{X} / L_{B}$ (Figs. 2] and [5 respectively) demonstrate a characteristic rise and fall of X-ray emission. The peak of this emission is coincident with the merger, a trend that is also seen observationally (Read \& Ponman 1998, their Fig. 13). One discrepancy may be the decline from the peak which is larger in the simulations than the observations. However, Figure 2] shows a wide range of peak luminosities depending on the presence of a $\mathrm{BH}$ and the metallicity of the gas. The luminosities will also fluctuate owing to the gas content of the progenitor disks as well as the merger orbit, complicating a quantitative comparison with the observations.

We can also compare the X-ray luminosity from the simulations subsequent to the merger to the study of disturbed ellipticals by O'Sullivan et al. (2001b). These authors determined that $L_{X} / L_{B}$ steadily increases with remnant age when age is measured by the fine structure parameter $\Sigma$ (Schweizer \& Seitzer 1992) and is zero at the last merger event. The slope of the best-fit relation by O'Sullivan et al. is shown as a short line in Figure 5 ("OFP01"). The shallowness of this X-ray evolution demonstrates that the increase of $L_{X} / L_{B}$ over time is a very subtle effect and that the hot gaseous halos of elliptical galaxies are grown over long periods of time.

For the simulation that includes $\mathrm{BHs}$, the $L_{X} / L_{B}$ ratio is nearly constant after the merger, a by-product of the equivalent fading of both $L_{X}$ and $L_{B}$. While we followed each remnant for only a short period of time after the merger and the isolated nature of the galaxies in our simulations make the long term luminosity evolution fairly uncertain, it does not appear that our remnant $L_{X} / L_{B}$ increases at the same rate as observed merger remnants.

\subsection{Remnant emission}

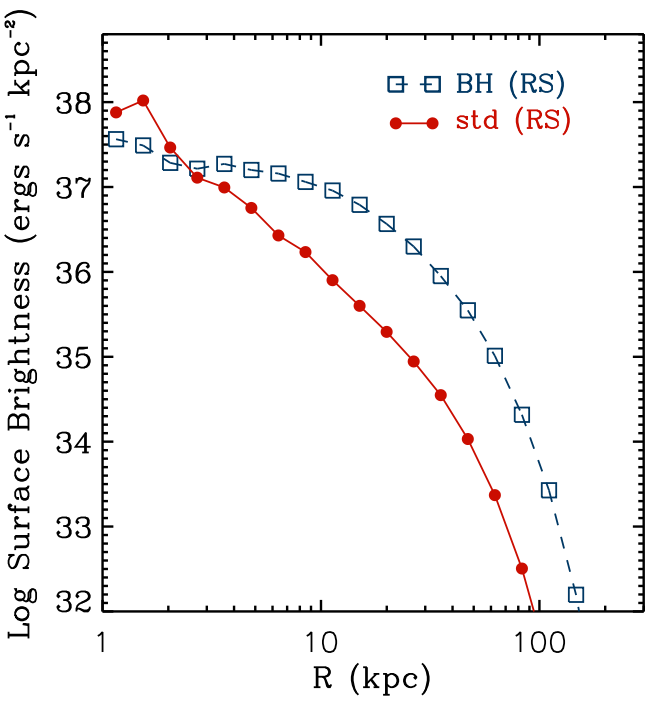

FIG. 6. - Azimuthally averaged X-ray surface brightness profile. The line types are identical to Fig. 5

As discussed in $\S$ पthe "merger hypothesis" posits that the interaction and merger of two spiral galaxies leads to the formation of an elliptical. In general, our simulations support this picture as we are left with a spheroidal stellar component surrounded by a halo of hot, X-rayemitting gas, similar to observed elliptical galaxies. In this section we investigate this in more detail by directly comparing the X-ray emission of our remnants to observations of post-merger and relaxed elliptical galaxies.

To begin, we show the X-ray surface brightness in Figure 6. The emission is calculated using the RS77 metalline cooling and is azimuthally averaged from 100 random viewing directions. The presence of $\mathrm{BHs}$ has a significant impact on the shape and radial extent of the X-ray emission. Apparently, energy injected by the BH decreases the emission on small scales $(\leq 3 \mathrm{kpc})$, and increases the emission on large scales $(\geq 3 \mathrm{kpc})$. In both cases, however, there is detectable emission to $\sim 100 \mathrm{kpc}$.

It is common practice to fit the observed X-ray surface brightness profiles of elliptical galaxies to a beta model of the form

$$
\Sigma_{X}(R)=\Sigma_{X, 0}\left[1+\left(R / R_{\text {core }}\right)^{2}\right]^{-3 \beta+0.5},
$$

where $\Sigma_{X, 0}$ is the central surface brightness, $R_{\text {core }}$ is the core radius, and $\beta$ quantifies the slope at large projected radii. We fit equation (3) to the surface brightness of our remnants and find the best fit to yield $\beta=1.85$ and $R_{\text {core }}=43.3 \mathrm{kpc}$ for the $\mathrm{BH}$ remnant and $\beta=2.12$ and $R_{\text {core }}=38.6 \mathrm{kpc}$ for the remnant without a $\mathrm{BH}$.

We note that the above fit was performed over the entire surface brightness profile, which includes emission out to $\sim 100 \mathrm{kpc}$. At these radii, the X-ray surface brightness is over 5 orders of magnitude fainter than the central regions. This large dynamic range is nearly 3 orders of magnitude more than is probed by the standard integration time of observations. Thus, a more realistic comparison can be achieved by restricting our fits to radii where the X-ray surface brightness is within 2 orders of magnitude of the peak. This corresponds to 12 and 50 $\mathrm{kpc}$ for the standard and $\mathrm{BH}$ remnant, respectively. In 


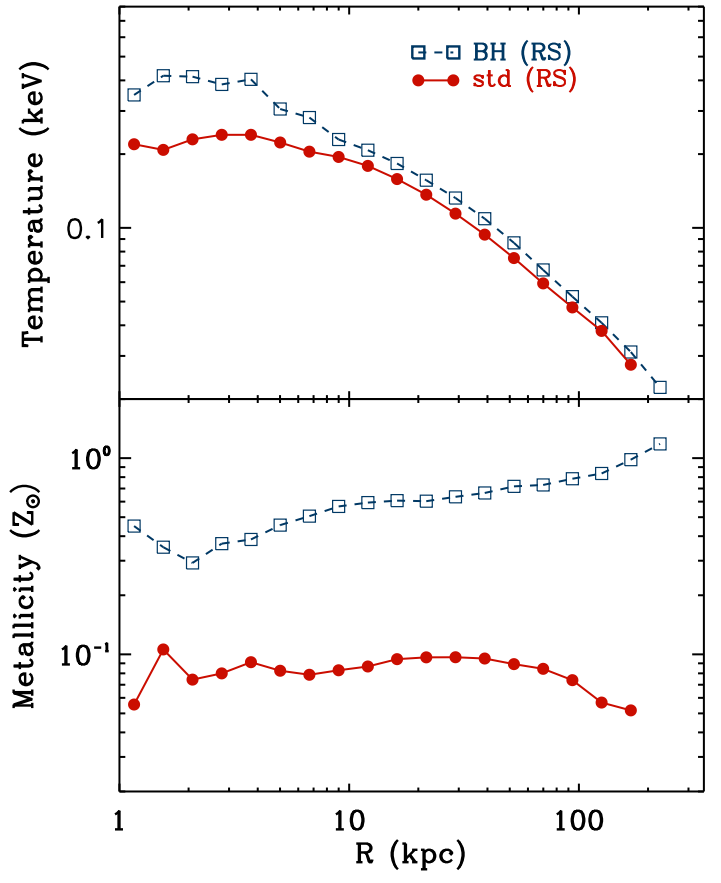

FIG. 7.- Projected temperature and metallicity profiles for the merger remnants. Both temperature and metallicity are X-ray luminosity weighted and azimuthally averaged. Line types are as in Fig. 5

this case, we find $\beta=0.80$ and $R_{\text {core }}=18.4 \mathrm{kpc}$ for the $\mathrm{BH}$ remnant and $\beta=0.53$ and $R_{\text {core }}=0.73 \mathrm{kpc}$ for the remnant without a $\mathrm{BH}$.

The $\beta$-profile fits restricted to the inner surface brightness profiles are in much better agreement with observations than the unrestricted fits. Observed merger remnants have measured $\beta$ values between 0.5 and 1.15 (Nolan et al. 2004; Fabbiano \& Schweizer 1995; Kim \& Fabbiano 2003). We also note the much flatter profile and larger core radius of the $\mathrm{BH}$ remnant. These features are observed in several X-ray faint early-type galaxies (O'Sullivan \& Ponman 2004b).

In addition to the X-ray surface brightness profile, an increasing number of galaxies have well determined temperature and metallicity profiles. Acquiring this information has been possible with the high spatial and spectral resolution of Chandra and $X M M-$ Newton, as well as the proper accounting of point sources and complex modeling of the hot X-ray emitting plasma. In particular, recent work has shown that multi-temperature plasma models provide a much better fit to the data and result in a larger estimate for the hot gas metallicity (Buote \& Fabian 1998).

Figure 7 shows the temperature (top) and metallicity (bottom) profiles for our merger remnants. Regardless of the presence of $\mathrm{BHs}$, the temperature is approximately isothermal within $\sim 10 \mathrm{kpc}$, and a strongly decreasing function at larger radii. The additional energy injected by the $\mathrm{BH}$ uniformly heats the gas at all radii without affecting the slope. However, inside of $\sim 2.5 \mathrm{kpc}$, the temperature difference is much larger owing to different cooling times of the hot gas. Without a BH, gas has a shorter cooling time and a cool(er) core has formed. In contrast, the energy input by the $\mathrm{BH}$ generates a hot(ter) cusp. We note that these curves are expected to have some time variability since gas has cooling times less than the Hubble time in both instances (see $\S 3.5$.

In comparison to the temperature, the differences in the metallicity profiles are much more striking. As described earlier, the $\mathrm{BH}$ induced galactic wind is "massloaded" and transports a significant amount of metals to large radii. This metal-rich wind results in an increasing metallicity profile. The largest radial bin is supersolar and the most metal rich. As we showed in $\S[3.2$ the trend of increasing metallicity with radius continues to the unbound wind material as well. Without a BH, metals are retained very near to where they are produced and hence the metallicity profile is flat or slightly decreases.

The majority of the spatially resolved data exists for $\mathrm{X}$-ray luminous early-type galaxies. These systems typically have nearly isothermal temperature profiles and metallicity gradients, although there is very often a slight decrease in temperature at small or large radii, or both (O'Sullivan \& Ponman 2004a b; Sivakoff et al. 2004; Kim \& Fabbiano 2003; Mushotzkv et al. 1994). At first glance, the profiles presented in Figure 7 appear to be at odds with the observed trends. However, as we mentioned previously, restricting our analysis to the inner regions would render the temperature profile much closer to isothermal. The metallicity gradient may be indicative of mixing between metal-rich gas produced during the merger and diffuse low-metallicity gas that is accreted by the galaxy, a process that is not included in the present simulations. We also note that X-ray luminous galaxies are generally large ellipticals, and thus our comparison is best directed toward merger remnants, which, because they are X-ray faint, do not usually have measured temperature or metallicity profiles.

One generic feature of observed ellipticals and merger remnants is the near solar metallicity of $\mathrm{X}$ ray gas (Nolan et al. 2004; Kim \& Fabbiand 2003; Humphrev \& Buote 2006). This is generally reproduced in the simulations that include a BH. Because the no-BH merger produces an X-ray halo of low metallicity, this suggests a mechanism to produce the low metallicities observed in a few X-ray faint ellipticals (O'Sullivan \& Ponman 2004b). This could occur if black hole growth is inefficient, as would be the case if the progenitors are gas-poor, or if the emission is so faint that one could only detect the central part of the rising metallicity profile present in the $\mathrm{BH}$ remnant.

\subsection{Spherical Profiles}

Figure 8 shows the spherically averaged gas density (top), cooling time (middle), and entropy (bottom) for $\mathrm{X}$-ray gas and provides a general picture of the state of hot gas in the merger remnants. While the remnant profiles are quite similar regardless of the presence of $\mathrm{BHs}$, there is a distinct transition at $\sim 10 \mathrm{kpc}$. Inside of this radius, gas in the no- $\mathrm{BH}$ remnant has a higher density, and thus a significantly shorter cooling time, and lower entropy. Outside of this radius, the trend reverses, mirroring what was seen in the X-ray surface brightness profiles in Figure 6.

It is noteworthy that the temperature and metallicity profiles shown in Figure 7 do not show a break. In this case, the $\mathrm{BH}$ remnant has a higher temperature and metallicity at all radii. Together, these trends suggest 


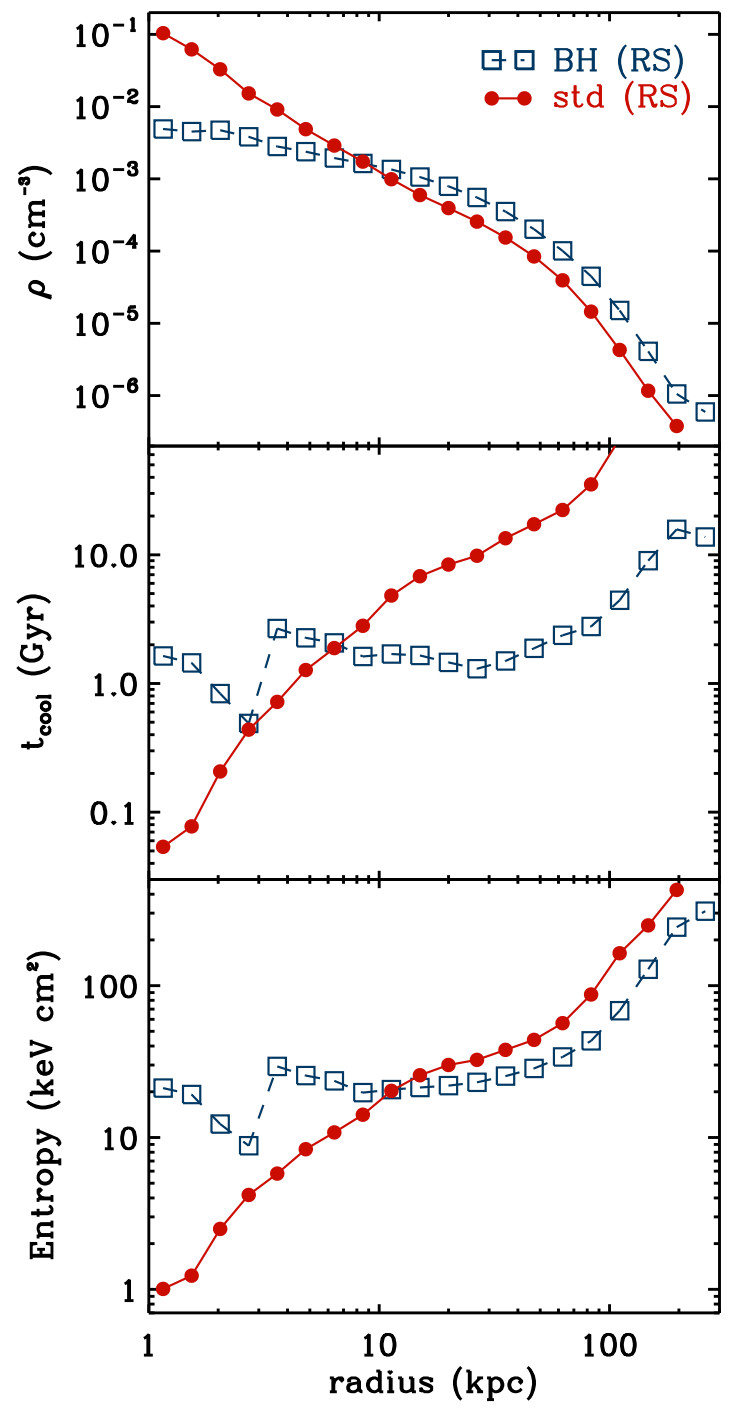

FIG. 8.- Spherically averaged density, cooling time, and entropy for X-ray emitting gas. Line types are as in Fig. 5

that feedback from the BH has pumped energy and metals into the gaseous component and affected diffuse gas at all radii. In response to this injection of energy, gas flows outward (and some even escapes in a wind), filling more of the dark matter halo than when no $\mathrm{BH}$ is present.

Even though gas that is located at $>10 \mathrm{kpc}$ is relatively similar in temperature and density in both the $\mathrm{BH}$ and no-BH cases, there is a significant difference in the cooling times, caused by the higher metallicity of gas in the $\mathrm{BH}$ remnant. In fact, much of the existing X-ray halo would cool within the next several Gyr in this model.

Finally, the spherical gas profiles display kinks at $\sim$ $3 \mathrm{kpc}$. While very pronounced in the cooling time and entropy profiles, this kink exists in some form in all the profiles, both projected and spherical. Features such as this are quite common in the simulations with $\mathrm{BHs}$ and result from a small number of residual cold gas clumps within the inner few kpc.

\section{SCALING WITH PROGENITOR GAS FRACTION AND} SIZE

Because hot gas is generated by shocks that attend the disk-galaxy collision, and is bolstered by feedback from black hole accretion, it is natural to expect that the Xray emission will depend on the amount of gas present in the progenitor disk galaxies as well as their masses. To understand this relationship better, we perform a series of merger simulations that span a range of progenitor gas fractions and masses as well as the orbits by which the disk galaxies merge.

All the progenitor disk galaxies used for this parameter exploration have identical halo properties such as concentration, spin parameter, and mass fraction. While holding these properties constant, we systematically change the disk gas fraction or virial velocity, or both. In total, we construct three halos smaller $\left(V_{200}=56\right.$, 80 , and $\left.115 \mathrm{~km} \mathrm{~s}^{-1}\right)$ than our fiducial model $\left(V_{200}=\right.$ $\left.160 \mathrm{~km} \mathrm{~s}^{-1}\right)$ and three that are larger $\left(V_{200}=225,320\right.$, and $500 \mathrm{~km} \mathrm{~s}^{-1}$ ). These virial velocities correspond to total masses spanning $5.8 \times 10^{10}-4.2 \times 10^{13} M_{\odot}$, a range of nearly 3 orders of magnitude. For each galaxy the exponential disk composes $4.1 \%$ of the total mass, with a fixed fraction $f$ in a collisional gaseous component. The compound galaxies are realized with 500,000 particles to represent the dark matter and 50,000 to represent the disk. Since the disk comprises both stellar and gaseous components, a fraction $f \times 50,000$ are considered gas and the remainder represent the collisionless stellar disk. In what follows we investigate four values of $f, 0.05,0.2$, 0.4 , and 0.8. Black holes are included in all of the simulations described in this section and the X-ray emission is calculated using the metallicity-dependent RS77 model.

Before we begin our exploration of the effects of varying the progenitor mass and gas fraction, we wish to determine if the fully co-planar progenitor disk orientations and radial orbit of our fiducial disk-galaxy merger significantly affect the resultant X-ray luminosity, discussed at length in $\S 3$ To this end, we merged the identical progenitor disks on the fiducial orbit with three alternate disk orientations. Besides our fiducial co-planar orientation $\left(\phi_{1}=\phi_{2}=\theta_{1}=\theta_{2}=0\right)$, we also simulated a tilted orientation $\left(\phi_{1}=30, \theta_{1}=60, \phi_{2}=-30, \theta_{2}=45\right)$, another tilted orientation $\left(\phi_{1}=-109, \theta_{1}=-30, \phi_{2}=\right.$ $\left.71, \theta_{2}=-30\right)$, and a polar orientation $\left(\phi_{1}=60, \theta_{1}=\right.$ $60, \phi_{2}=150, \theta_{2}=0$ ), where $\phi$ and $\theta$ are the standard spherical coordinates, and the subscripts 1 and 2 denote the two progenitor disks. The X-ray evolution for these runs is presented in the left panel of Figure 9 In the right panel of this figure, we show the X-ray evolution for our fiducial progenitor disk and fiducial orientation merging on six orbits with different angular momenta, one with less and five with more.

Together, the orbits and orientations presented in Figure 9 display a range of X-ray luminosities. At any particular time, $L_{X}$ may differ by over an order of magnitude. Much of this variability is attributable to the effect that orbits and orientations have on the consumption of gas, and thus the effective gas fraction at the time the galaxies interact. An additional factor is the growth of the black hole, as larger black holes will input more energy and produce additional hot gas.

To understand how the progenitor gas fraction influ- 

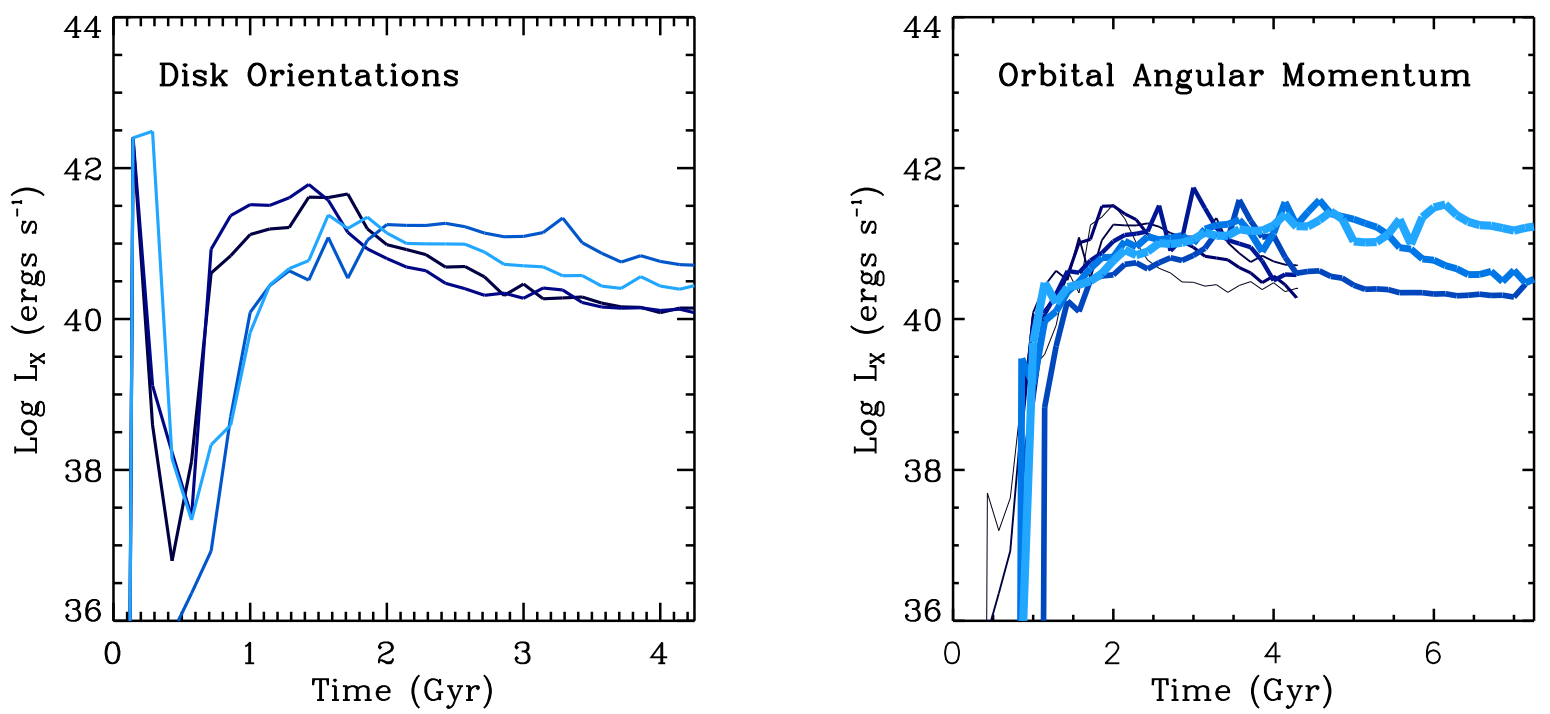

FIG. 9.- X-ray luminosity calculated using the metallicity-dependent RS77 model for several disk galaxy major mergers. In all cases, the progenitor disks are identical, while their orientation with respect to the orbital plane (left), or their merging orbit (right) has been varied.

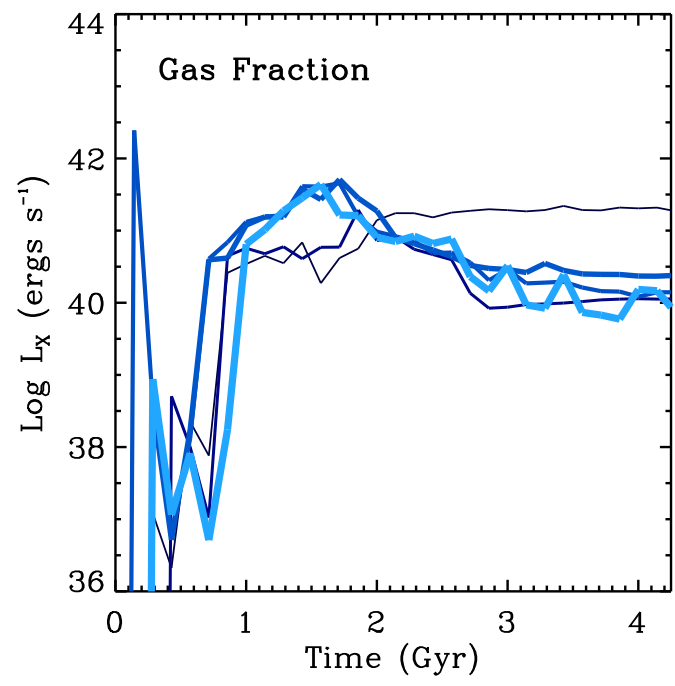

FIG. 10.- X-ray luminosity (RS77) during a major merger between disks with varying progenitor gas fractions. The color and thickness of each line is proportional to the gas fraction, with lighter, thicker lines representing the high gas fraction mergers and dark thin lines the low gas fraction mergers.

ences the X-ray luminosity without the complications associated with various orbits and orientations, we resimulate the fiducial merger while systematically varying the progenitor gas fraction. The resultant X-ray evolution is presented in Figure 10 As the progenitor gas fraction increases, the peak X-ray luminosity also increases. There is also an increase in $L_{X}$ prior to the merger, from $T=1.0$ to $1.8 \mathrm{Gyr}$, owing to the larger BHs and additional hot gas produced by their feedback. Because the $\mathrm{BH}$ feedback drives a powerful wind in the high gas fraction mergers, the X-ray luminosity drops subsequent to the peak. There is very little difference in the remnant

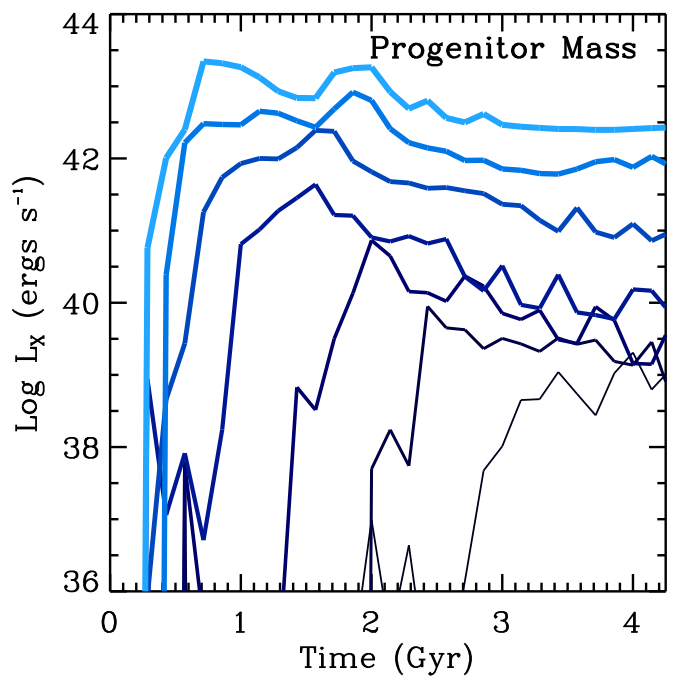

FIG. 11.- Shown is the X-ray luminosity (RS77) during disk galaxy major mergers for progenitors of varying mass, from $5.8 \times 10^{10} M_{\odot}$ to $4.2 \times 10^{13} M_{\odot}$. The mergers between more massive galaxies produce larger X-ray luminosities and these curves are drawn with progressively lighter, and thicker, lines.

X-ray emission for gas fractions greater than $20 \%$.

For the lowest gas fraction progenitor in Figure 10 the $\mathrm{X}$-ray emission is very small prior to the final merger. Many of these trends with gas fraction can be understood in terms of the feedback provided by the $\mathrm{BH}$ driving a large scale wind. As the gas fraction (and black hole mass) increase the wind becomes dominant and affects a larger amount of gas. Thus, the temperature and entropy of the remnant gas steadily increase in remnants with larger progenitor gas fractions. These effects will be the subject of future work that details the winds produced by black hole feedback (Hopkins et al., in preparation). 
The last dependency we explicitly address is progenitor mass. For this, we merge galaxies with a variety of masses on orbits that are self-similar, i.e. the pericentric distance is scaled in proportion to the size of each disk. Figure 11presents the X-ray evolution during these mergers. In all instances, the progenitor gas fraction was $80 \%$.

There is a regular progression for more massive progenitors to produce more X-ray emission. This is to be expected, if gas settles into hydrostatic equilibrium in each remnant halo. There is also a subtle difference between the maximum X-ray luminosity and the remnant $\mathrm{X}$-ray luminosity. Both the most and least massive galaxies have relatively little difference between the maximum and remnant $L_{X}$, while moderate masses have a large difference. This trend results from two competing effects. First, the impact of black hole feedback varies with system mass in a differential manner (Hopkins et al. 2006b), being increasingly more violent for more massive galaxies. Second, the gas consumption is much more efficient for more massive systems (Robertson et al. 2005) and thus they have lower effective gas fractions.

Finally, we summarize the production of X-rays and hot gas during the various disk-galaxy major mergers by compiling a number of global quantities for each merger remnant. In order to characterize the X-ray luminosity we determine the peak and remnant $L_{X}$ for each galaxy merger. We also find the mass of gas that composes the hot X-ray halo and the mass of gas that becomes unbound from the galaxy potential owing to the black hole feedback-induced blowout. These global quantities are shown in Figure 12 for our entire merger series, which includes the seven different mass initial conditions, four initial disk gas fractions, and four disk orientations. The scatter resulting from the four initial disk orientations simulated is shown by the $1 \sigma$ error bars for all quantities.

The top panels of Figure 12 demonstrate what was also apparent in Figure 11 namely, that more massive galaxy mergers produce more X-ray luminosity. For a self-similar model we expect $L_{X} \sim \sigma^{4}$, which is close to what is present in the simulated remnants. There is, however, a slight flattening at high $\sigma$, which reflects the more efficient star formation and thus less relative gas in these systems.

Apparent in the middle panels of Figure 12 is a trend for low-mass mergers to produce more unbound material. This is opposite to the trend found for X-ray gas mass, where the most massive systems have a larger fraction. Both trends hold across all progenitor gas fractions investigated. However, there is a systematic increase in the fraction of unbound gas with progenitor gas fraction. This trend reflects the increased wind efficiency induced by the black hole feedback for these systems.

The bottom panels of Figure 12 show the metallicity of the X-ray and unbound gas. In nearly all the remnants, both the unbound and X-ray gas have solar or supersolar metallicity. There is a slight decrease in metallicity at high masses, as these systems have very short gas consumption timescales (Robertson et al. 2005). Thus many of the metals become locked up in the stars, producing a correlation between remnant mass and stellar metallicity.

\section{DISCUSSION}

The previous section demonstrates that galaxy mergers produce significant $\mathrm{X}$-ray emission and that each merger remnant contains hot, X-ray emitting gas. However, we have not addressed whether this X-ray emission is comparable to observed ellipticals. Observations indicate that merger remnants are underluminous when compared with elliptical galaxies and that ellipticals build their X-ray emission slowly, over the course of several Gyr (O'Sullivan et al. 2001b). In this section we compare our merger remnants to common scaling relations for elliptical galaxies and speculate on their future evolution.

\subsection{The $L_{X}-L_{B}$ relation}

One of the most common relations used in the study of X-ray emission from individual galaxies is the $L_{X^{-}}$ $L_{B}$ relation. Figure 13 shows our entire series of gas fraction and size/mass remnants (from Fig. 12) in the $L_{X}-L_{B}$ plane. Also shown on this plot as points are data from O'Sullivan et al. (2001a) and luminous ellipticals from O'Sullivan et al. (2003) as crosses. The dotdashed line (with the shallower slope) that demarcates the lower bound of observed galaxies is an estimate of the point source contribution to the total X-ray luminosity (Ciotti et al. 1991). Galaxies that reside near this line have X-ray emission that is presumably dominated by point sources. Shown with a dashed line of slope $\sim 2.7$ is the best-fit relation for luminous ellipticals from O'Sullivan et al. (2003).

Our merger remnants trace out an arc that passes through the observed galaxies. Because we have not included any contribution to the X-ray luminosity from point sources, the low-mass remnants fall well below the observations. This is consistent with the expectation that these systems radiate little diffuse gas emission or that the diffuse gas is too cold to be easily detected. Our moderate $L_{B}\left(\sim 10^{10}-10^{11}\right)$ galaxies are very comparable to observed ellipticals, while the higher $L_{B}$ systems are underluminous.

The comparison between observed ellipticals and our remnants is muddled by two facts, one is the uncertain $L_{B}$ owing to the unknown star formation history of stars that are formed before we begin our simulation, and the second is the baryon fraction of our initial disk galaxies, which we assume is less than one-third of the cosmic mean.

To address the first point, we assume that stars present at the beginning of the simulation have been steadily formed during the $5 \mathrm{Gyr}$ prior to our $T=0$. Metallicities are selected randomly between solar and 1/10 solar and $L_{B}$ is calculated using the models of Bruzual \& Charlot (2003). We also note that the stopping point for our simulations is arbitrary. An additional 5 Gyr of evolution would decrease $L_{B}$ by another factor of 2 .

The second point above, the initial baryon fraction, is particularly relevant for our most massive systems, whose closest analogs are probably groups rather than individual galaxies. Groups tend to have baryon fractions closer to the cosmic mean value (Sanderson et al. 2003 ) and thus it may not be surprising that these remnants are underluminous. We also find it intriguing that $\mathrm{X}$-ray emission is only detected in groups that have at least one early-type galaxy (Mulchaev et al. 2003). This correlation naturally arises when the X-ray emission and 


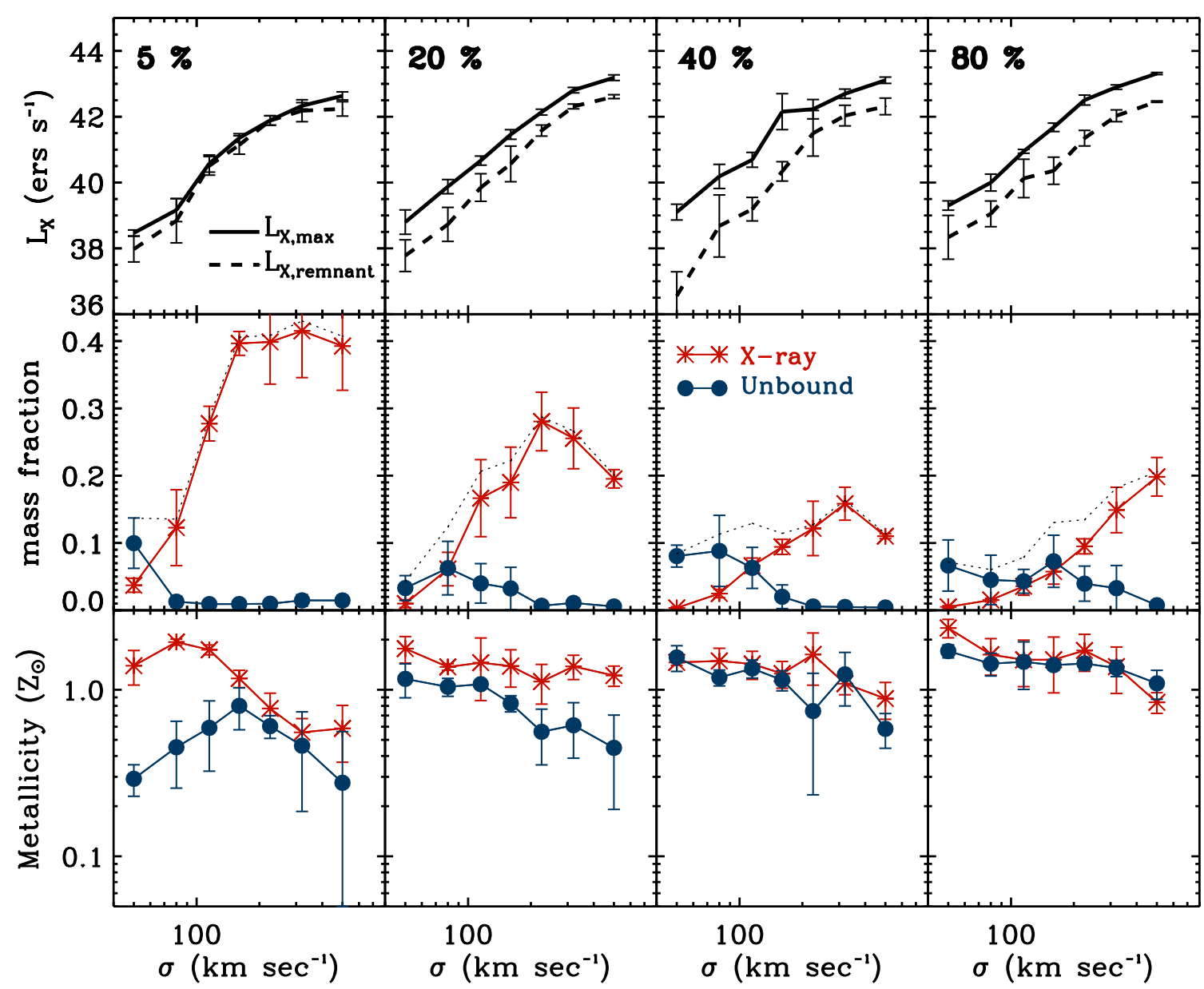

FIG. 12.- Global properties of the hot gas produced during disk-galaxy major mergers which include an accreting BH and its associated feedback. Each column shows results for mergers with identical progenitor gas fractions, indicated in the top panel of each column, for a variety of progenitor masses, as measured by the central stellar velocity dispersion of the remnants. The top row shows the maximum and remnant (RS77) X-ray luminosity during the merger. The middle row shows the fraction of the progenitor gas that is either physically unbound (i.e., $E>0$ ) from the remnant or that constitutes the hot X-ray halo. The bottom row shows the average metallicity of the unbound wind and X-ray gas.

early-type galaxy are formed in a common process, as we suggest here.

\subsection{The $L_{X}-T_{X}$ relation}

Figure 14 shows the remnant X-ray luminosity versus X-ray-weighted average temperature for our merger remnants in the $L_{X}-T_{X}$ plane. The $L_{X}-T_{X}$ relation is commonly used to study X-ray emission from clusters, groups, and more recently, elliptical galaxies. The bestfit relation for ellipticals appears to have an offset in both slope and normalization from the relations found for groups and clusters (which themselves are not identical), indicating that these systems have had an injection of energy (or entropy) above that expected from purely self-similar gravitational collapse (see Voit 2005, for a review). On this figure we also plot the data of O'Sullivan et al. (2003), which consists primarily of luminous ellipticals, as crosses, and their best-fit relation which has a slope of 4.8 .

The majority of the remnants are overluminous when compared to the observed $L_{X}-T_{X}$ relation, while the most massive remnants are underluminous. The only remnants that appear to match the observed data well are the remnants of mergers between disks with $80 \%$ initial gas fractions.

The discrepancy for the most massive remnants appears to be that they are simply underluminous, as this is the case for $L_{X}-T_{X}$ as well as $L_{X}-L_{B}$ H்owever, most of the other merger remnants appear consistent with the observed $L_{X}-L_{B}$ relation, and thus it is unclear whether they are really overluminous or whether their temperature is underestimated. The latter scenario could arise because of the radial temperature gradient present in most of the simulations. Hence, using a smaller aperture to measure the X-ray temperature or only detecting the central emission will yield higher $T_{X}$ than that measured here.

\subsection{The $\sigma-T_{X}$ relation}

As a final comparison, we plot the X-ray temperature against the stellar central velocity dispersion in Figure 15] As discussed in $\S 5.2 T_{X}$ is the mean X-ray weighted gas temperature and, as shown in $\S 3.4$ is a decreasing function of radius. The central stellar velocity dispersion is measured within the half-mass radius from 100 random projections. The typical $1 \sigma$ error owing to 


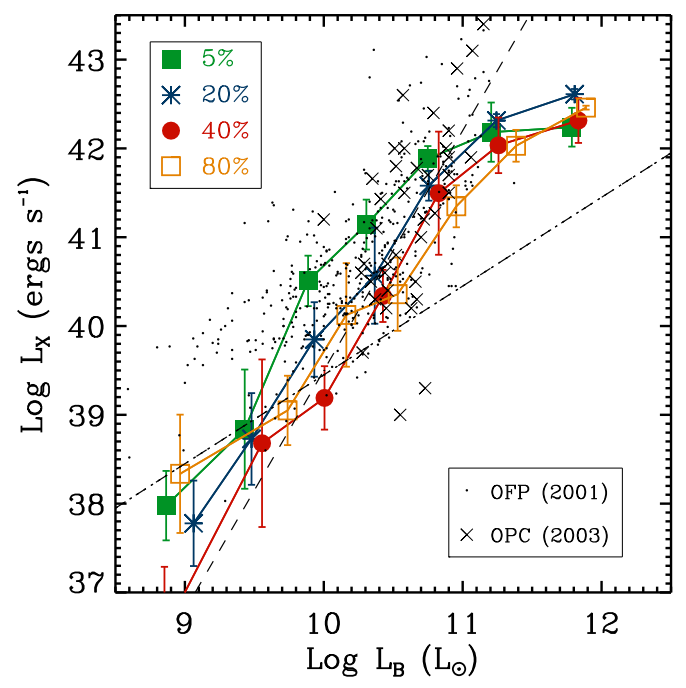

FIG. 13.- Merger remnants from our entire series of gas fraction and size/mass simulations plotted in the $L_{X}-L_{B}$ plane. These are the same systems as in Fig. 12 The black points are galaxies from the X-ray catalog of O'Sullivan et al. (2001a, OFP01), and the crosses are X-ray luminous ellipticals from O'Sullivan et al. (2003, OPC03). The dotted line is the estimated point source contribution $\log L_{\text {discrete }}=29.5 \log L_{B}$ from Ciotti et al. (1991), and the dashed line is the best-fit relation for luminous ellipticals found by OPC03.

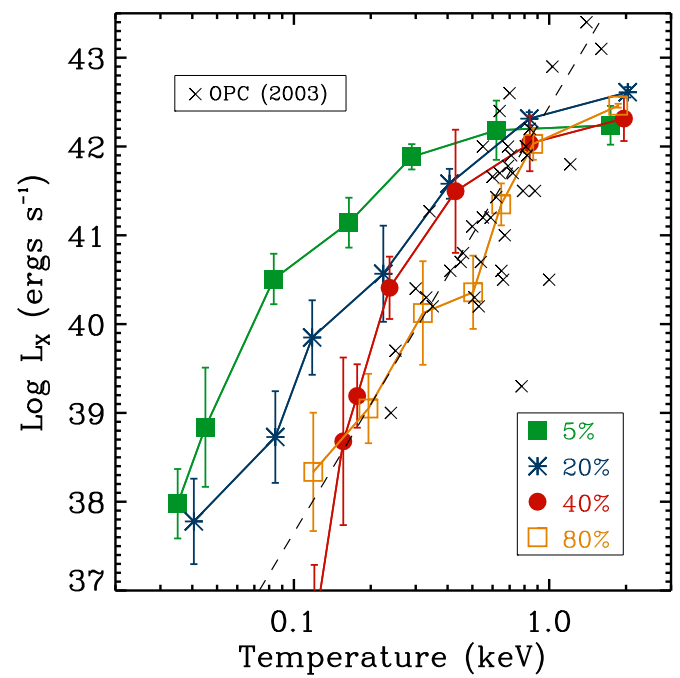

Fig. 14. - Bolometric X-ray luminosity vs. X-ray-weighted average temperature for our merger remnants. The crosses are $\mathrm{X}$ ray-luminous ellipticals from OPC03, along with their best-fitting line.

projection effects is $\sim 10 \%$.

Provided that both the gas and stars are relaxed and in equilibrium, both $\sigma$ and $T_{X}$ are a measure of the system potential. Also shown in Figure 15 is a dashed line expected when the gas and stars are in equipartition, i.e.,

$$
\mu m_{\mathrm{p}} \sigma^{2}=k T,
$$

where $\mu$ is the mean mass per particle, and $m_{\mathrm{p}}$ is the proton mass.

Figure [15] plots $\sigma$ versus $T_{X}$ for our series of merger remnants. In general, there exists a tight relation between $\sigma$ and $T_{X}$ for high mass remnants, signifying that

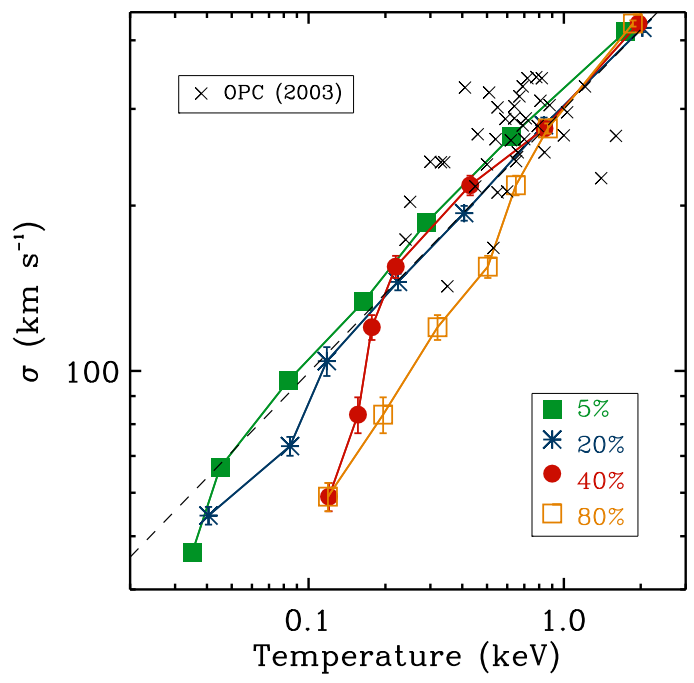

Fig. 15. - Merger remnants and the central stellar velocity dispersion $\sigma$ vs. $T_{X}$ plane. The crosses are X-ray-luminous ellipticals from OPC03. The dashed line marks equipartition of energy between X-ray gas and stellar velocity dispersion.

these systems contain gas that is in hydrostatic equilibrium throughout the remnant halo. There exists a trend for low-mass systems to peel away from the $\sigma-T_{X}$ relation. In particular, the remnants produced by gas-rich mergers show the largest discrepancy. These remnants are also strongly affected by the BH feedback (see, e.g., the amount of unbound gas mass in Fig. 12) and their high temperature reflects this additional energy input.

\subsection{Building an X-ray halo}

Overall, our model for the production of X-ray emission during the merger of two equal mass disk galaxies successfully matches the rise and fall of X-ray emission observed for the Toomre sequence (Read \& Ponman 1998). In addition, we find that the X-ray luminosity of the merger remnants span a wide range that depends on the initial disk gas fraction and size/mass. Compared to observed early-type galaxies of equivalent $L_{B}$, our models are broadly consistent with the data for $\log \left(L_{B}\right)<11 L_{\odot}$, and underluminous for more massive remnants. However, because the hot gas is generally consistent with the $L_{X}-T_{X}$ and $\sigma-T_{X}$ relations, we argue that a disk-galaxy merger that includes the feedback from accreting supermassive BHs is a sufficient mechanism to produce hot gas in the correct "state," i.e., metal-rich and in hydrostatic equilibrium, but in some cases there is simply not enough of this gas.

As stated in the introduction, three mechanisms have been proposed to explain the growth of a luminous Xray halo for elliptical galaxies, infalling tidal material, the reacquisition of material ejected in a galactic wind during the formation of the elliptical, and stellar mass loss. We discuss each of these in turn.

\subsubsection{Infalling tidal material}

One definitive conclusion from our simulations is that infalling tidal material contributes very little to the remnant X-ray emission. While tidal material continues to 
rain down on the remnant long ( $\sim 1 \mathrm{Gyr})$ after the nuclei have coalesced, the mass of these cold tidal tails is small $(\sim 20 \%)$ compared to the mass of hot diffuse gas already in place. Not only is the mass in the tidal material not sufficient to significantly alter the X-ray luminosity, but the efficiency at which this infalling cold gas is converted to X-ray-emitting hot gas is less than $30 \%$. Thus, the diffuse hot gas is largely in place soon after the merger, as demonstrated in the $T=1.85 \mathrm{Gyr}$ panel of Figure 1 and the tidal material serves only to "top off" the amount of hot gas. This is consistent with the theory that shells around ellipticals form from accreted tidal debris (Hernquist \& Spergel 1992), because the stellar luminosity of these features is small compared with that of the host galaxies.

\subsubsection{Re-acquisition of ejected hot gas}

Within the context of the simulations we present, only the mergers that include BHs and produce a wind have a reservoir of ejected gas to reacquire. This is evidenced by Figures 3 and 12, which show that the galactic wind is ubiquitous in small-mass halos and depends on the initial disk gas fraction. In some cases, the wind contains more mass than that which remains in the X-ray halo.

Of course, all of our remnants will also accrete pristine gas through the hierarchical growth of structure, which we do not follow here. This gas is likely to be acquired by infall from the ambient intergalactic medium (which may be pre-enriched at a low level) or via discrete subunits. However, the high metallicity observed in the halos of hot gas around local ellipticals (Humphrev \& Buote 2006) suggests that this is not the dominant mechanism to form the diffuse X-ray halo. Re-acquiring metal-enriched wind material allows for the growth of the X-ray halo while also maintaining a high metallicity. It is also possible that the enriched wind material will mix with the ambient intergalactic medium, thereby diluting its metallicity. Effects such as this may be responsible for the scatter in the observed metallicities of X-ray halos or in radial metallicity gradients.

\subsubsection{Stellar Mass Loss}

Owing to the large population of stars formed during the merger, we expect a significant contribution to the hot gas content from ongoing stellar mass loss. The generation of diffuse hot gas from stellar mass loss has been discussed extensively in the literature (see, e.g., Ciotti et al. 1991) and is generally punctuated by a brief period of outflows triggered by ongoing Type I supernovae and stellar mass loss, followed by an extended period $(\sim 10 \mathrm{Gyr})$ in which gas is retained, and the hot gaseous halo then builds up. We emphasize that this "wind" is in addition to and would follow the galactic wind induced by $\mathrm{BH}$ feedback. This long timescale for the build up of the X-ray halo is consistent with the observations of elliptical galaxies and merger remnants, as discussed by O'Sullivan et al. (2001a). While our merger simulations do not alter this picture, they do provide a physically motivated initial condition that includes the mass of young stars.

For the simulations we study here, the majority of the gas initially present in the progenitor galaxies is consumed by star formation. Thus the mass of young stars is typically 3 - 4 times that of the hot gas, which is either in the X-ray halo or unbound. While the amount of mass that is recycled from new stars back into the interstellar medium depends on the assumed initial mass function; if, for example, we assume a value of $\sim 25 \%$, then stellar mass loss will contribute an amount of hot gas that is roughly equivalent to what already exists from the merger alone.

\subsection{Galactic Winds}

A generic feature of the merger simulations that include feedback from an accreting black hole is the production of a large-scale outflow of metal-enriched gas. This outflow carries a significant fraction of the available gas mass and eventually deposits this gas throughout the halo and in some cases out of the galactic potential. Winds such as these may play a significant role in redistributing metals and may be required in order to terminate star formation and produce red ellipticals (Springel et al. 2005a; Hopkins et al. 2006c).

Observationally, there is evidence that winds such as these exist (for a review see Veilleux et al. 2005). At low redshift, winds are mainly observed in starburst galaxies via metal-line absorption and emission studies. It has been shown that there is a correlation between the outflow rate and the star formation rate and that some of these winds are powerful enough to escape the galaxy (Rupke et al. 2005a.b; Martin 2005). At high redshift, evidence for large-scale outflows comes from blueshifted absorption lines, redshifted Ly $\alpha$ emission lines (Pettini et al. 2002; Adelberger et al. 2003; Shaplev et al. 2003), and the excess of CIV absorption systems and a deficit of neutral hydrogen near Lyman break galaxies (Adelberger et al. 2005).

It is currently unclear how closely the winds produced in the simulations resemble observed winds. We note that understanding these winds in more detail may require embedding our simulations in a diffuse intergalactic medium, as many of the observations are actually a signature of the interaction between the wind and the local diffuse medium. This fact should be kept in mind when interpreting the fractional wind material presented in Figure 12 Theoretical models predict that all galaxy halos, including those of spirals, should contain some hot gas component, yet it is currently unclear if these hot gas halos are observed (Toft et al. 2002; Benson et al. 2000). In any case, including diffuse gas will provide a backstop against which the outflowing gas will interact, causing it to thermalize some of its energy, slow down, and possibly decrease the amount of outflowing material. We have performed one simulation in which both initial disks included a diffuse gaseous halo with one-quarter the baryonic mass. In this experiment the wind punched through the diffuse gas, and this resulted in very little difference from the case without any diffuse gas. More work will be necessary to determine how the wind interacts with diffuse gas and to compare these simulations to the observations.

As a final point, we note that the absence of these outflows in simulations without black holes does not mean that winds are only produced by accreting black hole feedback. This is quite apparent observationally, where systems such as M82 contain significant outflows and a corresponding starburst, but there is no evidence for an 
accreting black hole. In our simulations the outflows are produced by point-like energy injection and one could easily consider other formulations of star formation (see e.g., Springel \& Hernauist 2003) that would also produce galactic outflows.

\section{SUMMARY AND CONCLUSIONS}

Our hydrodynamic simulations show that collisions between gas-rich disk galaxies generate appreciable X-ray emission. This emission comes from hot diffuse gas that is produced by strong shocks attending the merger process. The presence of an accreting black hole increases the X-ray luminosity during the merger through the production of a hot, metal-rich galactic wind. This galactic wind transports a significant fraction of metals from the galactic center to the diffuse gaseous halo and outside the potential well of the remnant galaxy.

The wind-induced metal enrichment appears to be necessary to reproduce the near solar metallicity of observed ellipticals (Humphrev \& Buote 2006). Transporting metals out of the galaxy potential well may also help explain the high metallicity of the intra-group and intracluster media and the apparent deficit of baryons in lower mass galaxies.

Our merger remnants span a wide range of luminosities, yet are broadly consistent with X-ray properties of elliptical galaxies. This result suggests that galaxy mergers are a viable mechanism for generating the observed hot gas present in most interacting and merging systems.
In addition, we argue that both stellar mass loss and the reacquisition of galactic wind material are viable mechanisms to increase the luminosity of the diffuse X-ray halo.

This paper shows that galaxy mergers may play an integral role in the production of the hot, metal-rich gaseous halos that are present in most luminous elliptical galaxies. Using simulations similar to ours, recent studies demonstrate that gas-rich galaxy mergers that include black hole accretion and feedback can reproduce the black hole mass- central velocity dispersion relation (Di Matteo et al. 2005) and the colors (Springel et al. 2005a), fundamental plane (Robertson et al.|2005), and kinematics of elliptical galaxies (Cox et al. 2005a). Together, these results provide strong evidence for a "cosmic cycle" (see Fig. 1 of Hopkins et al. 2006a) of galaxy formation and evolution, in which gas-rich galaxy mergers and the growth of supermassive black holes play key roles.

We thank Ewan O'Sullivan, Duncan Forbes, and Jesper Sommer-Larsen for useful comments, and the referee for suggestions that significantly improved this work. This work was supported in part by NSF grants ACI 96-19019, AST 00-71019, AST 02-06299, and AST 0307690, NASA ATP grants NAG5-12140, NAG5-13292, and NAG5-13381. The simulations were performed at the Center for Parallel Astrophysical Computing at the Harvard-Smithsonian Center for Astrophysics.

\section{REFERENCES}

Adelberger, K. L., Shapley, A. E., Steidel, C. C., Pettini, M., Erb, D. K., \& Reddy, N. A. 2005, ApJ, 629, 636

Adelberger, K. L., Steidel, C. C., Shapley, A. E., \& Pettini, M. 2003, ApJ, 584, 45

Barnes, J. E. 1992, ApJ, 393, 484

Barnes, J. E. \& Hernquist, L. 1996, ApJ, 471, 115

Benson, A. J., Bower, R. G., Frenk, C. S., \& White, S. D. M. 2000 MNRAS, 314, 557

Bruzual, G. \& Charlot, S. 2003, MNRAS, 344, 1000

Buote, D. A. \& Fabian, A. C. 1998, MNRAS, 296, 977

Ciotti, L., Pellegrini, S., Renzini, A., \& D'Ercole, A. 1991, ApJ, 376,380

Cox, T. J., Dutta, S., Di Matteo, T., Hernquist, L., Hopkins, P. F., Robertson, B., \& Springel, V. 2005a, ApJ submitted

Cox, T. J., Jonsson, P., Primack, J., \& Somerville, R. S. 2005b, MNRAS, submitted (astro-ph/0503201)

Cox, T. J., Primack, J., Jonsson, P., \& Somerville, R. S. 2004, ApJ, 607, L87

Di Matteo, T., Springel, V., \& Hernquist, L. 2005, Nature, 433, 604

Fabbiano, G. \& Schweizer, F. 1995, ApJ, 447, 572

Fabbiano, G., Zezas, A., \& Murray, S. S. 2001, ApJ, 554, 1035

Hernquist, L. 1990, ApJ, 356, 359

-. 1992, ApJ, 400, 460

-. 1993a, ApJ, 404, 717

-. 1993b, ApJ, 409, 548

Hernquist, L. \& Quinn, P. J. 1988, ApJ, 331, 682

- 1989, ApJ, 342, 1

Hernquist, L. \& Spergel, D. N. 1992, ApJ, 399, L117

Hernquist, L. \& Weil, M. L. 1992, Nature, 358, 734

Hopkins, P. F., Hernquist, L., Cox, T. J., Di Matteo, T., Robertson, B., \& Springel, V. 2006a, ApJS, 163, 1

-. 2006b, ApJ, 639, 700

Hopkins, P. F., Hernquist, L., Cox, T. J., Robertson, B., \& Springel, V. 2006c, ApJS, 163, 50

Hopkins, P. F., Hernquist, L., Martini, P., Cox, T. J., Robertson, B., Di Matteo, T., \& Springel, V. 2005d, ApJ, 625, L71

Humphrey, P. J. \& Buote, D. A. 2006, ApJ, 639, 136

Huo, Z. Y., Xia, X. Y., Xue, S. J., Mao, S., \& Deng, Z. G. 2004 , ApJ, 611, 208
Jenkins, L. P., Roberts, T. P., Ward, M. J., \& Zezas, A. 2005, MNRAS, 357, 109

Jog, C. J. \& Solomon, P. M. 1992, ApJ, 387, 152

Kim, D. \& Fabbiano, G. 2003, ApJ, 586, 826

Malin, D. F. \& Carter, D. 1983, ApJ, 274, 534

Martin, C. L. 2005, ApJ, 621, 227

Mathews, W. G. \& Brighenti, F. 2003, ARA\&A, 41, 191

McDowell, J. C., Clements, D. L., Lamb, S. A., Shaked, S., Hearn, N. C., Colina, L., Mundell, C., Borne, K., Baker, A. C., \& Arribas, S. 2003, ApJ, 591, 154

Mihos, J. C. \& Hernquist, L. 1996, ApJ, 464, 641

Mulchaey, J. S., Davis, D. S., Mushotzky, R. F., \& Burstein, D. 2003, ApJS, 145, 39

Mushotzky, R. F., Loewenstein, M., Awaki, H., Makishima, K., Matsushita, K., \& Matsumoto, H. 1994, ApJ, 436, L79

Naab, T. \& Burkert, A. 2003, ApJ, 597, 893

Navarro, J. F., Frenk, C. S., \& White, S. D. M. 1995, MNRAS, 275,720

Nolan, L. A., Ponman, T. J., Read, A. M., \& Schweizer, F. 2004, MNRAS, 353, 221

O'Sullivan, E., Forbes, D. A., \& Ponman, T. J. 2001a, MNRAS, 328,461

一. 2001b, MNRAS, 324, 420

O'Sullivan, E. \& Ponman, T. J. 2004a, MNRAS, 354, 935

-. 2004b, MNRAS, 349, 535

O'Sullivan, E., Ponman, T. J., \& Collins, R. S. 2003, MNRAS, 340, 1375

Pettini, M., Rix, S. A., Steidel, C. C., Adelberger, K. L., Hunt, M. P., \& Shapley, A. E. 2002, ApJ, 569, 742

Quinn, P. J. 1984, ApJ, 279, 596

Raymond, J. C. \& Smith, B. W. 1977, ApJS, 35, 419

Read, A. M. 2003, MNRAS, 342, 715

-. 2005, MNRAS, 359, 455

Read, A. M. \& Ponman, T. J. 1998, MNRAS, 297, 143

Robertson, B., Cox, T. J., Hernquist, L., Franx, M., Di Matteo, T., Hopkins, P. F., Martini, P., \& Springel, V. 2005, ApJ, 641, 21

Robertson, B., Yoshida, N., Springel, V., \& Hernquist, L. 2004, ApJ, 606, 32

Rupke, D. S., Veilleux, S., \& Sanders, D. B. 2002, ApJ, 570, 588 -. 2005a, ApJ, 632, 751 
-. 2005b, ApJS, 160, 115

Sanderson, A. J. R., Ponman, T. J., Finoguenov, A., Lloyd-Davies, E. J., \& Markevitch, M. 2003, MNRAS, 340, 989

Schweizer, F. 1980, ApJ, 237, 303

Schweizer, F. \& Seitzer, P. 1992, AJ, 104, 1039

Schweizer, F., Seitzer, P., Faber, S. M., Burstein, D., Dalle Ore, C. M., \& Gonzalez, J. J. 1990, ApJ, 364, L33

Shapley, A. E., Steidel, C. C., Pettini, M., \& Adelberger, K. L. 2003, ApJ, 588, 65

Sivakoff, G. R., Sarazin, C. L., \& Carlin, J. L. 2004, ApJ, 617, 262

Smith, B. J., Struck, C., \& Nowak, M. A. 2005, AJ, 129, 1350

Springel, V. 2000, MNRAS, 312, 859

-. 2005, MNRAS, 364, 1105

Springel, V., Di Matteo, T., \& Hernquist, L. 2005a, ApJ, 620, L79 . 2005b, MNRAS, 361, 776

Springel, V. \& Hernquist, L. 2002, MNRAS, 333, 649
-. 2003, MNRAS, 339, 289

Strickland, D. K., Heckman, T. M., Weaver, K. A., Hoopes, C. G., \& Dahlem, M. 2002, ApJ, 568, 689

Toft, S., Rasmussen, J., Sommer-Larsen, J., \& Pedersen, K. 2002, MNRAS, 335, 799

Toomre, A. 1977, in Evolution of Galaxies and Stellar Populations, p.401

Toomre, A. \& Toomre, J. 1972, ApJ, 178, 623

Veilleux, S., Cecil, G., \& Bland-Hawthorn, J. 2005, ARA\&A, 43, 769

Voit, G. M. 2005, Rev. Mod. Physics, 77, 207

Weil, M. L. \& Hernquist, L. 1993, ApJ, 405, 142

Xia, X. Y., Xue, S. J., Mao, S., Boller, T., Deng, Z. G., \& Wu, H. 2002, ApJ, 564, 196 